\title{
Daily Low Dose of Erythropoietin and Neuroinflammation
}

\section{Reza Nejat ${ }^{1}$, Ahmad Shahir Sadr ${ }^{2,3,4}$, Alireza Ebrahimi ${ }^{5}$, Alireza Nabati ${ }^{6}$, Elham Eshaghi $^{7}$}

${ }^{1}$ Anesthesiologist, Fellow of Critical Care Medicine, Former Assistant Professor, Anesthesia and Critical Care Medicine Dept., Shahid Beheshti University of Medical Sciences, Tehran, IRAN. rezanejat@yahoo.com, rezanejat.com

${ }^{2}$ Department of Computer Science, Faculty of Mathematical Sciences, Shahid Beheshti University, Tehran, Iran,

${ }^{3}$ Department of Phytochemistry, Medicinal Plants and Drugs Research Institute, Shahid Beheshti University, Tehran, Iran,

${ }^{4}$ School of Biological Sciences, Institute for Research in Fundamental Sciences (IPM), Tehran, Iran

${ }^{5}$ Anesthesiologist, Subspecialist of Critical Care Medicine, Chief of ICU, Shohadaye Gomnam Hospital, Shahid Beheshti University of Medical Sciences, Tehran, Iran.

${ }^{6}$ Neurosurgeon, AJA University of Medical Sciences, Tehran, Iran

${ }^{7}$ Elham Eshaghi, Resident in Anesthesiology Program, AJA University of Medical Sciences, Tehran, Iran

\section{Summary}

Neuroinflammation, defined as inflammatory reactions mediated by cytokines, chemokines, reactive oxygen species, and secondary messengers in the central nervous system (CNS) including the brain and spinal cord is the basis of many neurological disorders ${ }^{[1]}$ Recently, erythropoietin (EPO) has been considered and studied as a modulator of neuroinflammation. ${ }^{[2-4]}$ On this article minireview of pathophysiology of neuroinflammation and the neuroprotective effects of EPO is discussed and a case of subacute huge subdural hematoma with double mydriasis operated urgently, treated with low daily dose (vs high dose once or twice a month in the literature) of EPO and recovered fully and discharged home with good consciousness is reported. In addition, the probable outcome of erythropoietin administration in patients with neuroinflammation in COVID19 is considered.

\section{Introduction}

Neuroinflammation has a deleterious effect on the CNS in a time- and severity-dependent fashion; short mild form of it may be considered as rather repairing which contributes to the neurodevelopment, neuroprotection and neuroplasticity, yet its severe prolonged version may be debilitating to the patients. ${ }^{[5,6]}$ This phenomenon, with its energy-consuming metabolic- 
demanding nature has been linked to the secondary pathological changes elicited in hypoglycemia [7], the ischemic brain disorders ${ }^{[8,9]}$, intracerebral hemorrhage ${ }^{[10,11]}$, traumatic brain injury (TBI) ${ }^{[12,13]}$, Alzheimer's disease ${ }^{[14-16]}$, Parkinson's disease ${ }^{[17,18]}$ and other neurodegenerative disorders such as amyotrophic lateral sclerosis (ALS) ${ }^{[19]}$, and multiple sclerosis (MS) ${ }^{[20,21]}$. It has recently been related to the neurobiological disorders like of epilepsy ${ }^{[22]}$, depression ${ }^{[23,}{ }^{24]}$, obsessivecompulsive disorder ${ }^{[25]}$ and schizophrenia ${ }^{[26]}$. There are lots of data in the literature showing that EPO could be an appropriate drug to subside neuroinflammation specially in traumatic brain injuries. The dosage of EPO used in these articles was reported to be between 10000 and 40000 units. ${ }^{[27,28]}$ As to the debating subjects associated with administration of EPO like hypertension and thrombophilia we decided to use lower doses of EPO (4000 units/day for 3 weeks or till a reasonable level of consciousness is observed) in our patients with neuroinflammatory disorders.

\section{Low Daily Dose of Erythropoietin in a Patient with Huge Subdural Hematoma}

\section{Prone to Brain Death}

A 56-year-old woman was admitted to emergency room of Bazarganan Hospital, Tehran, Iran in November 22, 2018 due to gradual loss of consciousness (GCS:11 on admission) after falling down and having been traumatized to the head about two weeks prior to admission. She had right-sided hemiparesis started the day of admission. Mild mental retardation and drug-controlled epilepsy could be seen on her past medical history since her early ages of life. Physical examination of other organs revealed nothing. Her GCS deteriorated soon after non-contrasted CT scan was done for her (figure 1A, 1B) which showed a huge rightward shift of the brain due to subacute pooling of blood in subdural area in the left hemisphere of the brain.

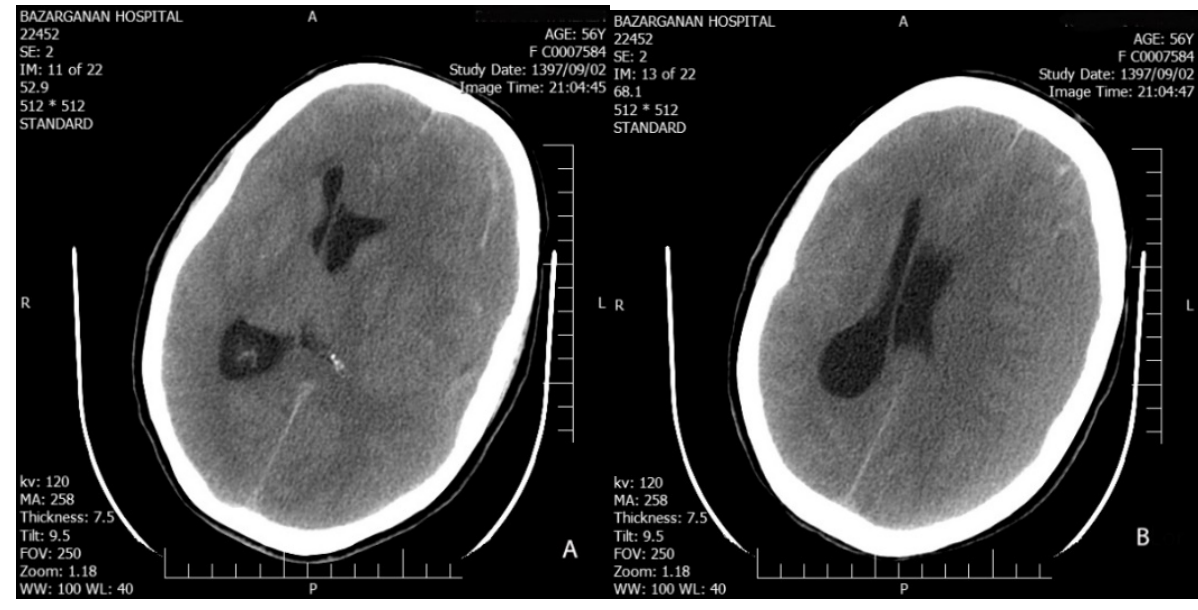

Figure 1A and 1B. Rightward huge shift of the brain due to subacute subdural hematoma 
As unilateral mydriasis occurred in the left pupil, the patient was sent to the operating room (OR) urgently to evacuate the blood after an open peripheral vein was secured and infusion of 1 liter of normal saline and loading dose of phenytoin were started. 8000 units of EPO was injected subcutaneously (SC), as well. Unilateral mydriasis turned to double mydriasis as she was put on the operating table. Operation terminated uneventfully and mydriasis turned to midsize pupils with sluggish reaction to the light as she arrived at ICU. Postop CT scan showed that the blood was evacuated successfully despite mild shift of the brain remained due to the edema of the left hemisphere (figure $2 \mathrm{~A}$ and $2 \mathrm{~B}$ ).

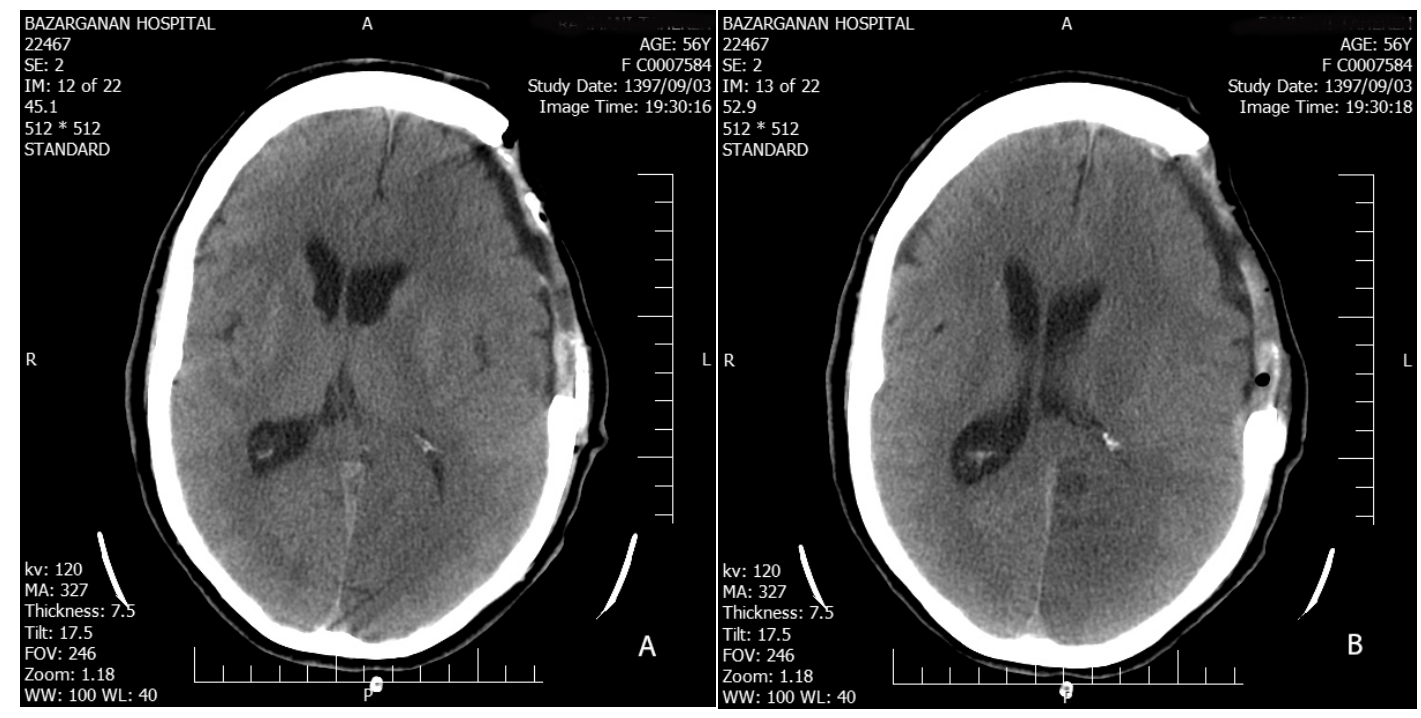

Figure $2 A$ and $2 B . P O D 1$ brain $C T$ scans

The patient was given 4000 units of SC EPO a day for the rest of her stay at ICU till reasonable recovering of her consciousness ( 2 weeks) with taking care that her hematocrit not rise to more than 33. No rise in the blood pressure or thrombotic vascular complication was seen in this period. MRI on the fifth post operation day (POD) showed ischemic lesions in the midbrain and left occipitotemporal area due to the pressure of the hematoma on these areas prior to operation (figure $3 \mathrm{~A}$ and $3 \mathrm{~B})$.

She started to show some sluggish motor reactions gradually and could open her eyes to some degree in December 1, 2018. Her consciousness improved day by day and her GCS reached to 14 in January 26, 2019 without any major neurologic deficit but some retardation in her speech and a mild degree of right-sided hemiparesis which improved within the next 3 months. 


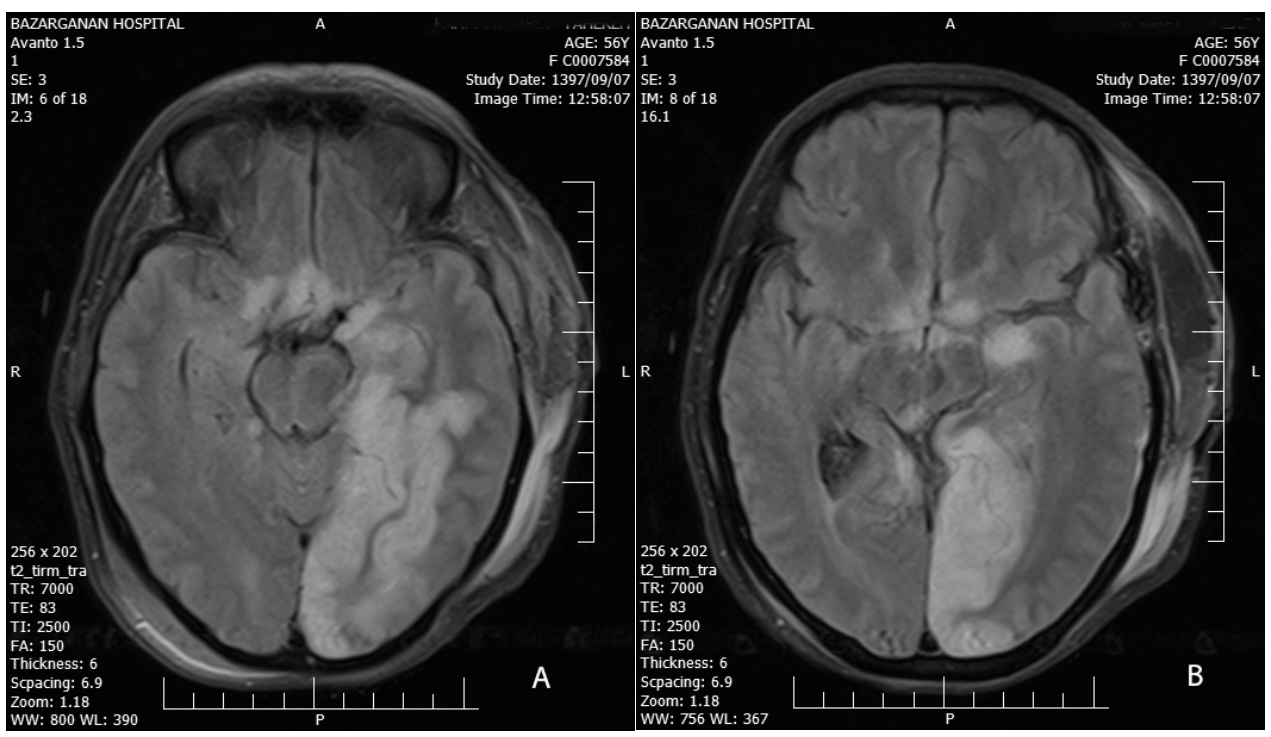

Figure $3 A$ and $3 B$. High intensity lesions in MRI in the midbrain and left occipitotemporal area; POD5

\section{Discussion}

Neuroinflammation is triggered by any type of stress to the nervous system. ${ }^{[29]}$ In this phenomenon astrocytes and microglia contribute as components of innate immunity to initiate cascade of synergistic effects of cytokines. Astrocytes, the most distributed glial cell type in the CNS, support the homeostasis of microenvironment of neural cells and regulate neurotransmitters and synaptic functions. Stimulation of astrocytes may morphologically and functionally turn them into two distinct forms: radial glial-like and reactive astrocytes. ${ }^{[30,31]}$ Radial glial-like astrocyte lineage represents mitogenically active multipotent stem cells in the adult brain which eventually constitute the source of neural cells and other astrocytes. ${ }^{[32,33]}$ Reactive astrocytes along with microglial, which change from $\mathrm{M}_{2}$ (anti-inflammatory) to $\mathrm{M}_{1}$ (pro-inflammatory) morphology, along with neural cells, with different innate immunity programs in different regions of the brain, contribute to defend against pathogens. ${ }^{[34,35]}$ These sentinel cells express pattern-recognition receptors (PRRs) including Toll-like receptors (TLR), NOD-like receptors (NLRs), receptor for advanced glycation end products (RAGE), and scavenger, complement and mannose receptors. $[6],[36-38]$

Brain cells can recognize pathogen-associated molecular patters (PAMPs) or host-derived danger/damage-associated molecular patterns (DAMPs) (heat shock proteins, ATP, S100B and 
HMGB) through their PRRs. ${ }^{[35],[39]}$ PAMPs and DAMPs activate signaling pathways such mitogen-activated protein kinases (MAPKs) and nuclear factor-kappa B (NF- $\kappa \mathrm{B})$. These pathways promote generation and rapid gene amplification of the inflammatroy cascade and expression of ICAM-1, VCAM-1, E-selection, and iNOS. ${ }^{[40-43]} \mathrm{NF}-\kappa \mathrm{B}$ pathway activation has been linked to neuroinflammatory responses in Parkinson's Disease, Alzheimer's Disease and other insults to the brain such as TBI and ischemic brain disorders. ${ }^{[44-46]}$ Furthermore, inhibition of NF- $\kappa$ B was shown to slow than the speed of progression of neurodegenerative disorders. ${ }^{[47]}$

Activation of astrocytes and microglia results in secretion of cytokines (IL-1 $\beta$, TNF- $\alpha$, and IL-6), $\alpha$-chemokines (MCP-1, MIP-1, and RANTES), and other inflammatory mediators such as cyclooxygenase-2 and MMP-9. ${ }^{[48-50]}$ On the other hand, MMPs are involved in the regulation and modification vascular endothelial growth factor (VEGF) in a positive feedback effect ${ }^{[51]}$. MMP inhibitors could halt releasing of mature TNF- $\alpha{ }^{[52]}$ Consistent with their abilities, MMPs, with proteolysis of the extracellular matrix proteins remained from inflammatory process, open the space to accommodate the inflammatory cells as well as newly produced blood vessels. Although as an anti-inflammatory effect, MMPs may modulate pro-inflammatory cytokines, the former's destructive potentials may damage the penumbra unless tissue inhibitors of matrix proteinase (TIMPs) come into action. ${ }^{[53,54]}$

Simultaneous occurrence of neuroinflammation and cell apoptosis in the CNS makes it difficult to uncover which one is the primary or secondary event. ${ }^{[55]}$ Moreover, blood brain barrier is disrupted in inflammatory reactions in the brain. This permits entry of inflammatory to the brain parenchyma. In this context, water content in the brain dysregulates as the function and distribution of aquaporins (AQPs) with constitutional pro-inflammatory effects are disturbed. These proteins are responsible for regulating transmembrane water transport, as well as some small molecules like glycerol. AQPs play a key role in astrocyte swelling and migration, function of BBB, and cytokine release, as well. ${ }^{[56,57]}$

Intriguingly, any type of the brain injury is associated with tissue hypoxia due to inflammation with high metabolic and oxygen demand, vascular injury and shortage of oxygen delivery. Relative oxygen deficiency and absolute hypoxic environment of acute phase of cerebral injury (6-12 hours post-injury period) induces expression of hypoxia inducible factor-1 $\alpha$ (HIF-1 $\alpha$ ) which promotes pro-apoptotic genes (BNIP3, NIX and NOXA). HIF-1 $\alpha$ in the genome binds with caspase 3 promoter which leads to apoptosis of the injured cells or in the penumbra. ${ }^{[58]}$ Furthermore, in the hypoxic environment, free radicals (ROS, RNS) generated by mitochondrial complex III induces 
both destruction of vital and structural molecules and stabilization of HIF-1 $\alpha .{ }^{[59,60]}$ In general, HIF-1 $\alpha$ is upregulated in normoxia (for example in post-reperfusion state) by some factors such as insulin-like growth factor-1 (IGF-1), thyroid hormone $\left(\mathrm{T}_{3}\right)$, cytokines (IL- $\beta$, IL-6, TGF- $\beta$, TNF$\alpha$ ), NFkB, free radicals (ROS, RNS), thrombin, PAMPs and DAMPs. ${ }^{[61-67]}$ The initial proapoptotic effect of HIF-1 $\alpha$ turns to pro-survival proteomes (like EPO, VEGF, glucose transporter-1, aldolase A, lactic dehydrogenase A, phosphofructokinase protein) after 48 hours. $^{[68,}$ ${ }^{69]}$ Accordingly, most of the hazardous effects of acute neuroinflammatory state is observed in the first few days. Thus, applying early anti-inflammatory/anti-apoptotic measure would be legitimate to decline the severity of the damage.

The beneficial effects of EPO on neuroinflammation have been discussed on a growing amount of literature in the last three decades. ${ }^{[70-73]}$ Fetal brain in rats exhibits high level of EPO receptor (EPO-R) which decreases after birth up to 100fold. EPO-R can be found on neurons, astrocytes and microglia in the adults, as well. It is implied that EPO, a growth hormone, is involved in the CNS development in fetal period and must govern some trophic and protective cascades in the brain in adulthood. ${ }^{[74,75]}$ It is worth to know that EPO could induce proliferation of cultured neuroprogenitor cell if added to the culture media. ${ }^{[76]}$ In an animal study EPO could promote differentiation of precursor cells to increase mature neurons and oligodendrocyte population in the hippocampus. ${ }^{[77]}$ Angiogenesis and neurogenesis in rats was shown to be induced if EPO is given in the first 24 hour of stroke. ${ }^{[78]}$ Anti-inflammatory/anti-apoptotic properties of (EPO) have long attracted the attention of experts. ${ }^{[79-81]}$ Arterial-thromboembolic induced brain ischemia treated with EPO in an animal study revealed limited neural loss and BBB disruption due to anti-apoptotic and anti-inflammatory potentials of EPO. ${ }^{\left[{ }^{82}\right]}$ Moreover, it has also been demonstrated that EPO could downregulate HIF-1 $\alpha$ expression in brain ischemia. ${ }^{[83]}$ As a significant finding, EPO was demonstrated to expand expression of its receptor (EPO-R), reduce the axonal damage, decline the level of IL- $\beta$, suppress neuroinflammation and increase sensorimotor and cognitive responses in an animal model and decrease traumatic axonal injury specially when the rat were kept hypoxic, as well. ${ }^{[72]}$

High-glucose induces apoptosis of retinal ganglionic cells which has been shown to be inhibited by EPO's stabilization of mitochondrial membrane potential. In this context, EPO could prevent releasing of cytochrome $\mathrm{C}$ and avoid upregulation of oxygen free radical and mitochondrial damage. ${ }^{[84]}$ Treatment with EPO upregulates mitochondrial complex III, IV and respiration and neural energetics. ${ }^{[85]}$ Similarly, EPO was found to be effective against neural cell apoptosis in 
glaucoma through PI-3-K/Akt pathway. ${ }^{[86]}$ This growth factor in rats saves microglia through its dose-dependent anti-apoptotic effect without disturbing their pro-inflammatory activities. Elevation of $\mathrm{Bcl} / \mathrm{Bax}$ ratio and prevention of caspase-3 and -9 are other EPO's abilities to survive microglia. ${ }^{[87-89]}$ In cell culture of murine microglia and astrocytes, EPO could protect astrocytes from oxidative stress injury and upregulate nitric oxide, while only exhibited antioxidant effects against ROS injury in microglia. ${ }^{[0]}$ EPO in brain injuries has been shown to inhibit AQP-4induced astrocyte swelling and to downregulate MMP-9, the latter through increasing the expression of TIMP-1 and upregulation of JAK-2/STAT3/STAT5 pathways. ${ }^{[91,92]}$

In a recent in vitro study, it was reported that plasma membrane of human $\mathrm{CD}^{+}$and $\mathrm{CD} 8^{+} \mathrm{T}$ cells contain EPO-R and EPO which could suppress alloreactive human T-cell immunity via inhibition of downstream T-cell and IL-2 receptor signaling pathways. ${ }^{[93]}$

Recently the potency of human recombinant type of EPO in treating neurodegenerative disorders has been considered. ${ }^{[94]}$ EPO has been effective in improvement of non-motor symptoms in Parkinson's disease and in preventing memory deficit by preserving hippocampal neurons in a rat model of Alzheimer's Disease. ${ }^{[95,96]}$ In drug-resistant depression, EPO has shown a promising effect. ${ }^{[97]}$ There are contradictory results regarding EPO efficacy in ALS, yet a new clinical trial has recently been conducted in South Korea. ${ }^{[98-100]}$

It should be noted that EPO should not be given to patients with primary and secondary nervous system neoplasia or any neoplasia in other organs because EPO as a pleiotropic growth factor with an anti-apoptotic property may cause spreading of the tumors, especially solid ones. ${ }^{[101]}$

\section{EPO and Neuroinflammation in Brain Involvement in COVID19}

In general, administration of this cytokine in sepsis and infections is a debating subject; on one side of this spectrum, macrophage function is suppressed in Salmonella infection in the presence of EPO. This suppressing ability of EPO against macrophages might be deleterious in sepsis. ${ }^{[102,}$ ${ }^{103]}$ On the other side it was uncovered that EPO could improve survival of mice in sepsis as it reverses irresponsiveness of the aorta to norepinephrine (NE), upregulates eNOS and downregulates iNOS. ${ }^{[104]}$ Neuroinflammation involving the brain in COVID19 needs special consideration. SARS-CoV2 follows the track of ACE2 in the brain. As to the proposed novel theory that is supported by a great amount of literature and attributes the pathophysiology of cytokine storm and inflammatory reactions in COVID19 to downregulation of ACE2 and subsequent hyperacute excess of angiotensin II (Ang II) relative to angiotensin(1-7) and supra- 
activation of angiotensin receptor type 1 (AT1R) ${ }^{[105]}$, EPO should be administered in these patients cautiously because of reciprocal positive interactions of EPO and Ang II. ${ }^{[106,107]}$

It has been reported that Ang II blood level in patients with COVID19 is higher than that in noninfected healthy people. ${ }^{[108]}$ AT1R located in brain circumventricular area and cerebrovascular endothelial cells is activated by circulating Ang II with the ability to impair neurovascular coupling and reduce cerebral blood flow (low dose of $0.1 \mathrm{pmol} / \mathrm{min}$ results in a $23 \%$ reduction). ${ }^{[109,110]}$ Local RAS has also been described in the brain, yet ACE2 was reported to predominate in the brain specially in the hypothalamus compared to angiotensin converting enzyme (ACE). ${ }^{[11]}$ ACE2 deficiency was shown to increase brain swelling and cell death in an animal model of brain ischemia. ${ }^{[112]}$ EPO exhibits synergistic effect on Ang II and NE in mobilization of intracellular $\mathrm{Ca}^{2+}$ in vascular smooth muscle cells which may last for about 60 minutes. ${ }^{[107]}$ Furthermore, EPO dose-dependently promotes expression of transient receptor potential canonical gene (TRPC) and TRPC channel protein up to 70\%. Ang II induces TRPC-mediated $\mathrm{Ca}^{2+}$ current which soars up significantly in the presence of EPO. ${ }^{[113]} \mathrm{Ca}^{2+}$ though mediates many homeostatic surviving pathways, its intracellular concentration is tightly regulated as increased sustained intracellular calcium content induces programmed cell death. ${ }^{[14]}$ Thus EPO in synergism with Ang II might induce apoptosis.

\section{Conclusion}

As the aforementioned patient's brain MRI shows ischemic lesions in the midbrain and occipitotemporal areas, it seems that intracranial pressure prior to operation was high enough to make these areas bloodless and prolong coma or even give the patient the vegetative state, postoperatively. The patient's double mydriasis on the operating room was a clue that brain parenchyma herniation was an imminent event. As the patient received routine neuroprotective medical care but it was just low dose of EPO that was added, and a great percentile of similar patients' outcome is not favorable, it is justifiable to regard EPO as the drug that could help the brain recover fast and flawlessly despite this vast ischemic lesion in the midbrain and occipitotemporal areas. As the issue of safety of EPO is debating due to its thrombophilic, hypertensive and inducing TRPC properties [113],[115-117], we use EPO with loading dose of 8000 units and maintenance dose of about 4000 units EPO a day for 3 weeks or less till consciousness recovers reasonably. In this period, we take care that hematocrit does not rise more than 33 . 
It seems that low regular daily dose of EPO compared to doses found in the literature (1000040000 units once or twice a month) is promising in treating neuroinflammatory disorders in the future with less complications.

\section{Acknowledgement:}

The authors would like to extend their greatest thanks to all the anesthesiologists and personnel of Bazarganan ICU, specially adorable nurses who always take care of the patients meticulously and to the radiologists and personnel of imaging department of this hospital.

\section{References:}

1. DiSabato, D.J., N. Quan, and J.P. Godbout, Neuroinflammation: the devil is in the details. Journal of neurochemistry, 2016. 139: p. 136-153.

2. Shin, T., et al., Erythropoietin and autoimmune neuroinflammation: lessons from experimental autoimmune encephalomyelitis and experimental autoimmune neuritis. Anatomy \& cell biology, 2012. 45(4): p. 215-220.

3. Moransard, M., et al., Erythropoietin reduces experimental autoimmune encephalomyelitis severity via neuroprotective mechanisms. Journal of neuroinflammation, 2017. 14(1): p. 202.

4. Chong, Z.Z., J.-Q. Kang, and K. Maiese, Apaf-1, Bcl-xL, cytochrome c, and caspase-9 form the critical elements for cerebral vascular protection by erythropoietin. Journal of Cerebral Blood Flow \& Metabolism, 2003. 23(3): p. 320-330.

5. Sochocka, M., B.S. Diniz, and J. Leszek, Inflammatory response in the CNS: friend or foe? Molecular neurobiology, 2017. 54(10): p. 8071-8089.

6. Shastri, A., D.M. Bonifati, and U. Kishore, Innate immunity and neuroinflammation. Mediators of inflammation, 2013. 2013.

7. Ratter, J.M., et al., Proinflammatory effects of hypoglycemia in humans with or without diabetes. Diabetes, 2017. 66(4): p. 1052-1061.

8. Fang, M., et al., Effect of Inflammation on the Process of Stroke Rehabilitation and poststroke depression. Frontiers in Psychiatry, 2019. 10.

9. Liu, R., et al., Role of neuroinflammation in ischemic stroke. Neuroimmunol Neuroinflammation, 2017. 4: p. 158-66.

10. Tschoe, C., et al., Neuroinflammation after intracerebral hemorrhage and potential therapeutic targets. Journal of stroke, 2020. 22(1): p. 29.

11. Mracsko, E. and R. Veltkamp, Neuroinflammation after intracerebral hemorrhage. Frontiers in cellular neuroscience, 2014. 8: p. 388.

12. Wofford, K.L., D.J. Loane, and D.K. Cullen, Acute drivers of neuroinflammation in traumatic brain injury. Neural regeneration research, 2019. 14(9): p. 1481.

13. Dinet, V., K.G. Petry, and J. Badaut, Brain-immune interactions and neuroinflammation after traumatic brain injury. Frontiers in neuroscience, 2019. 13: p. 1178.

14. Hensley, K., Neuroinflammation in Alzheimer's disease: mechanisms, pathologic consequences, and potential for therapeutic manipulation. Journal of Alzheimer's disease, 2010. 21(1): p. 1-14.

15. Calsolaro, V. and P. Edison, Neuroinflammation in Alzheimer's disease: current evidence and future directions. Alzheimer's \& Dementia, 2016. 12(6): p. 719-732.

16. Millington, C., et al., Chronic neuroinflammation in Alzheimer's disease: new perspectives on animal models and promising candidate drugs. BioMed research international, 2014. 2014. 
17. Wang, Q., Y. Liu, and J. Zhou, Neuroinflammation in Parkinson's disease and its potential as therapeutic target. Translational Neurodegeneration, 2015. 4(1): p. 19.

18. Gelders, G., V. Baekelandt, and A. Van der Perren, Linking neuroinflammation and neurodegeneration in Parkinson's disease. Journal of immunology research, 2018. 2018.

19. Liu, J. and F. Wang, Role of neuroinflammation in amyotrophic lateral sclerosis: cellular mechanisms and therapeutic implications. Frontiers in Immunology, 2017. 8: p. 1005.

20. Koudriavtseva, T. and C. Mainero, Neuroinflammation, neurodegeneration and regeneration in multiple sclerosis: intercorrelated manifestations of the immune response. Neural regeneration research, 2016. 11(11): p. 1727.

21. Al-Badri, G. and A. Castorina, Insights into the Role of Neuroinflammation in the Pathogenesis of Multiple Sclerosis. Journal of Functional Morphology and Kinesiology, 2018. 3(1): p. 13.

22. Vezzani, A., Epilepsy and Inflammation in the Brain: Overview and Pathophysiology: Epilepsy and Inflammation in the Brain. Epilepsy currents, 2014. 14(2_suppl): p. 3-7.

23. Jeon, S.W. and Y.K. Kim, Neuroinflammation and cytokine abnormality in major depression: cause or consequence in that illness? World journal of psychiatry, 2016. 6(3): p. 283.

24. Troubat, R., et al., Neuroinflammation and depression: A review. European Journal of Neuroscience, 2020.

25. Gerentes, M., et al., Obsessive-Compulsive Disorder: Autoimmunity and Neuroinflammation. Current psychiatry reports, 2019. 21(8): p. 78.

26. Aricioglu, F., et al., Neuroinflammation in schizophrenia: a critical review and the future. Klinik Psikofarmakoloji Bülteni-Bulletin of Clinical Psychopharmacology, 2016. 26(4): p. 429-437.

27. Liu, W.-C., et al., Therapeutic effect of erythropoietin in patients with traumatic brain injury: a meta-analysis of randomized controlled trials. Journal of neurosurgery, 2016. 127(1): p. 8-15.

28. Nirula, R., et al., Safety and efficacy of erythropoietin in traumatic brain injury patients: a pilot randomized trial. Critical care research and practice, 2010. 2010.

29. Yatsiv, I., et al., Erythropoietin is neuroprotective, improves functional recovery, and reduces neuronal apoptosis and inflammation in a rodent model of experimental closed head injury. The FASEB journal, 2005. 19(12): p. 1701-1703.

30. Banjara, M. and C. Ghosh, Sterile neuroinflammation and strategies for therapeutic intervention. International journal of inflammation, 2017. 2017.

31. Noctor, S.C., et al., Neurons derived from radial glial cells establish radial units in neocortex. Nature, 2001. 409(6821): p. 714-720.

32. Barry, D.S., J.M. Pakan, and K.W. McDermott, Radial glial cells: key organisers in CNS development. The international journal of biochemistry \& cell biology, 2014. 46: p. 76-79.

33. Lima, C.M.d., et al., Differential change in hippocampal radial astrocytes and neurogenesis in shorebirds with contrasting migratory routes. Frontiers in neuroanatomy, 2019. 13: p. 82.

34. Cho, H., et al., Differential innate immune response programs in neuronal subtypes determine susceptibility to infection in the brain by positive-stranded RNA viruses. Nature medicine, 2013. 19(4): p. 458.

35. Ising, C. and M.T. Heneka, Functional and structural damage of neurons by innate immune mechanisms during neurodegeneration. Cell death \& disease, 2018. 9(2): p. 1-8.

36. González-Reyes, R.E. and M.G. Rubiano, Astrocyte's RAGE: More than just a question of mood. Central Nervous System Agents in Medicinal Chemistry (Formerly Current Medicinal ChemistryCentral Nervous System Agents), 2018. 18(1): p. 39-48.

37. Kigerl, K.A., et al., Pattern recognition receptors and central nervous system repair. Experimental neurology, 2014. 258: p. 5-16.

38. Kong, Y. and Y. Le, Toll-like receptors in inflammation of the central nervous system. International immunopharmacology, 2011. 11(10): p. 1407-1414. 
39. Ransohoff, R.M. and M.A. Brown, Innate immunity in the central nervous system. The Journal of clinical investigation, 2012. 122(4): p. 1164-1171.

40. Liu, T., et al., NF-KB signaling in inflammation. Signal transduction and targeted therapy, 2017. 2(1): p. 1-9.

41. Rui, W., et al., PM2. 5-induced oxidative stress increases adhesion molecules expression in human endothelial cells through the ERK/AKT/NF-KB-dependent pathway. Journal of Applied Toxicology, 2016. 36(1): p. 48-59.

42. Sun, B., et al., Activation of NF $K B$ and Expression of ICAM-1 in Ischemic-reperfused Canine Myocardium. Journal of molecular and cellular cardiology, 2001. 33(1): p. 109-119.

43. Milstone, D.S., et al., Differential role of an NF-KB transcriptional response element in endothelial versus intimal cell VCAM-1 expression. Circulation research, 2015. 117(2): p. 166-177.

44. Singh, S.S., et al., NF-KB-Mediated Neuroinflammation in Parkinson's Disease and Potential Therapeutic Effect of Polyphenols. Neurotoxicity Research, 2019: p. 1-17.

45. Ju Hwang, C., et al., NF-KB as a Key Mediator of Brain Inflammation in Alzheimer's Disease. CNS \& Neurological Disorders-Drug Targets (Formerly Current Drug Targets-CNS \& Neurological Disorders), 2019. 18(1): p. 3-10.

46. Harari, O.A. and J.K. Liao, NF-KB and innate immunity in ischemic stroke. Annals of the New York Academy of Sciences, 2010. 1207: p. 32.

47. Li, Y., O. Sibon, and P. Dijkers, Inhibition of NF-KB in astrocytes is sufficient to delay neurodegeneration induced by proteotoxicity in neurons. Journal of neuroinflammation, 2018. 15(1): p. 261.

48. Bertheloot, D. and E. Latz, HMGB1, IL-1 $\alpha, I L-33$ and S100 proteins: dual-function alarmins. Cellular \& molecular immunology, 2017. 14(1): p. 43-64.

49. $\mathrm{Xu}, \mathrm{J}$, et al., Microglial activation induced by the alarmin S10OB is regulated by poly (ADP-ribose) polymerase-1. Glia, 2016. 64(11): p. 1869-1878.

50. Shih, R.-H., C.-Y. Wang, and C.-M. Yang, NF-kappaB signaling pathways in neurological inflammation: a mini review. Frontiers in molecular neuroscience, 2015. 8: p. 77.

51. Hollborn, M., et al., Positive feedback regulation between MMP-9 and VEGF in human RPE cells. Investigative ophthalmology \& visual science, 2007. 48(9): p. 4360-4367.

52. McGeehan, G.M., et al., Regulation of tumour necrosis factor- $\alpha$ processing by a metalloproteinase inhibitor. Nature, 1994. 370(6490): p. 558-561.

53. Cox, J.H. and C.M. Overall, Cytokine substrates: MMP regulation of inflammatory signaling molecules, in The Cancer Degradome. 2008, Springer. p. 519-539.

54. Arpino, V., M. Brock, and S.E. Gill, The role of TIMPs in regulation of extracellular matrix proteolysis. Matrix Biology, 2015. 44: p. 247-254.

55. Murphy, F., et al., Immunological Approaches to Prevent Neuronal Apoptosis During Neuroinflammation. Current Medicinal Chemistry-Anti-Inflammatory \& Anti-Allergy Agents, 2005. 4(4): p. 367-381.

56. Meli, R., C. Pirozzi, and A. Pelagalli, New perspectives on the potential role of aquaporins (AQPs) in the physiology of inflammation. Frontiers in physiology, 2018. 9: p. 101.

57. Tourdias, T., et al., Differential aquaporin 4 expression during edema build-up and resolution phases of brain inflammation. Journal of Neuroinflammation, 2011. 8(1): p. 143.

58. Van Hoecke, M., et al., Evidence of HIF-1 functional binding activity to caspase-3 promoter after photothrombotic cerebral ischemia. Molecular and Cellular Neuroscience, 2007. 34(1): p. 40-47.

59. Chandel, N.S., et al., Reactive oxygen species generated at mitochondrial complex III stabilize hypoxia-inducible factor- $1 \alpha$ during hypoxia a mechanism of $\mathrm{O} 2$ sensing. Journal of Biological Chemistry, 2000. 275(33): p. 25130-25138.

60. Klimova, T. and N. Chandel, Mitochondrial complex III regulates hypoxic activation of HIF. Cell Death \& Differentiation, 2008. 15(4): p. 660-666. 
61. Haibara, A.S., N.M. Sharma, and K.P. Patel, Hypoxia-Inducible Factor (HIF)-1 Alpha Expression Induced by Proinflammatory Cytokines in NG108-15 Neuronal Cells. The FASEB Journal, 2016. 30(1_supplement): p. 1234.3-1234.3.

62. Otto, T. and J. Fandrey, Thyroid hormone induces hypoxia-inducible factor $1 \alpha$ gene expression through thyroid hormone receptor $6 /$ retinoid $x$ receptor $\alpha$-dependent activation of hepatic leukemia factor. Endocrinology, 2008. 149(5): p. 2241-2250.

63. Qutub, A.A. and A.S. Popel, Reactive oxygen species regulate hypoxia-inducible factor $1 \alpha$ differentially in cancer and ischemia. Molecular and cellular biology, 2008. 28(16): p. 5106-5119.

64. Gorlach, A., et al., Thrombin activates the hypoxia-inducible factor-1 signaling pathway in vascular smooth muscle cells: role of the p22 phox-containing NADPH oxidase. Circulation research, 2001. 89(1): p. 47-54.

65. Feldser, D., et al., Reciprocal positive regulation of hypoxia-inducible factor $1 \alpha$ and insulin-like growth factor 2. Cancer research, 1999. 59(16): p. 3915-3918.

66. Jiang, F., et al., The role of insulin-like growth factor I and hypoxia inducible factor $1 \alpha$ in vascular endothelial growth factor expression in type 2 diabetes. Annals of Clinical \& Laboratory Science, 2013. 43(1): p. 37-44.

67. Yoshida, T., et al., Transcriptional upregulation of HIF-1 $\alpha$ by NF-K B/p65 and its associations with 6-catenin/p300 complexes in endometrial carcinoma cells. Laboratory investigation, 2013.93(11): p. 1184-1193.

68. Barteczek, P., et al., Neuronal HIF-1 $\alpha$ and HIF-2 $\alpha$ deficiency improves neuronal survival and sensorimotor function in the early acute phase after ischemic stroke. Journal of Cerebral Blood Flow \& Metabolism, 2017. 37(1): p. 291-306.

69. Chen, W., et al., Prodeath or prosurvival: two facets of hypoxia inducible factor-1 in perinatal brain injury. Experimental neurology, 2009. 216(1): p. 7-15.

70. Kumral, A., et al., Neuroprotective effect of erythropoietin on hypoxic-ischemic brain injury in neonatal rats. Neonatology, 2003. 83(3): p. 224-228.

71. Nejat, R., Erythropoietin and Neuroinflammation. Journal of Neuroscience and Neuropharmacology, 2019. 5: p. 36.

72. Hellewell, S.C., et al., Erythropoietin improves motor and cognitive deficit, axonal pathology, and neuroinflammation in a combined model of diffuse traumatic brain injury and hypoxia, in association with upregulation of the erythropoietin receptor. Journal of neuroinflammation, 2013. 10(1): p. 926.

73. Bond, W.S. and T.S. Rex, Evidence that erythropoietin modulates neuroinflammation through differential action on neurons, astrocytes, and microglia. Frontiers in immunology, 2014. 5: p. 523.

74. Olsen, N.V., Central nervous system frontiers for the use of erythropoietin. Clinical infectious diseases, 2003. 37(Supplement_4): p. S323-S330.

75. Nejat, R., Neuroprotective Effect of Erythropoietin. International Journal of Emergency Mental Health \& Human Resilience, 2018. 20: p. 21.

76. Alnaeeli, M., et al., Erythropoietin in brain development and beyond. Anatomy research international, 2012. 2012.

77. Hassouna, I., et al., Revisiting adult neurogenesis and the role of erythropoietin for neuronal and oligodendroglial differentiation in the hippocampus. Molecular psychiatry, 2016. 21(12): p. 17521767.

78. Wang, L., et al., Treatment of stroke with erythropoietin enhances neurogenesis and angiogenesis and improves neurological function in rats. Stroke, 2004. 35(7): p. 1732-1737.

79. Gawad, A., et al., Antiapoptotic properties of erythropoietin: novel strategies for protection of retinal pigment epithelial cells. Eye, 2009. 23(12): p. 2245-2250. 
80. Alural, B., et al., EPO mediates neurotrophic, neuroprotective, anti-oxidant, and anti-apoptotic effects via downregulation of miR-451 and miR-885-5p in SH-SY5Y neuron-like cells. Frontiers in immunology, 2014. 5: p. 475.

81. MacRedmond, R., G.K. Singhera, and D.R. Dorscheid, Erythropoietin inhibits respiratory epithelial cell apoptosis in a model of acute lung injury. European Respiratory Journal, 2009. 33(6): p. 14031414.

82. Thériault, P., et al., Sub-acute systemic erythropoietin administration reduces ischemic brain injury in an age-dependent manner. Oncotarget, 2016. 7(24): p. 35552.

83. Souvenir, R., et al., Erythropoietin inhibits HIF-1 $\alpha$ expression via upregulation of PHD-2 transcription and translation in an in vitro model of hypoxia-ischemia. Translational stroke research, 2014. 5(1): p. 118-127.

84. Wang, Y., et al., Erythropoietin (EPO) protects against high glucose-induced apoptosis in retinal ganglional cells. Cell biochemistry and biophysics, 2015. 71(2): p. 749-755.

85. Singhal, N., et al., Erythropoietin upregulates brain hemoglobin expression and supports neuronal mitochondrial activity. Molecular neurobiology, 2018. 55(10): p. 8051-8058.

86. Weishaupt, J.H., et al., Effect of erythropoietin axotomy-induced apoptosis in rat retinal ganglion cells. Investigative ophthalmology \& visual science, 2004. 45(5): p. 1514-1522.

87. Liao, Z., et al., Erythropoietin can promote survival of cerebral cells by downregulating Bax gene after traumatic brain injury in rats. Neurology India, 2009. 57(6): p. 722.

88. Sekiguchi, N., et al., Effect of erythropoietin on endothelial cell apoptosis induced by high glucose. Diabetes research and clinical practice, 2004. 66: p. S103-S107.

89. Wenker, S.D., et al., Protective action of erythropoietin on neuronal damage induced by activated microglia. The FEBS journal, 2013. 280(7): p. 1630-1642.

90. Pathipati, P. and D.M. Ferriero, The differential effects of erythropoietin exposure to oxidative stress on microglia and astrocytes in vitro. Developmental neuroscience, 2017. 39(1-4): p. 310322.

91. Tang, G. and G.-Y. Yang, Aquaporin-4: A potential therapeutic target for cerebral edema. International journal of molecular sciences, 2016. 17(10): p. 1413.

92. Souvenir, R., et al., Janus kinase 2 and tissue inhibitor of matrix metalloproteinase-1 mediate the protective effects of erythropoietin in in-vitro model of hypoxia ischemia. 2009, Federation of American Societies for Experimental Biology.

93. Cravedi, P., et al., Immunosuppressive effects of erythropoietin on human alloreactive $T$ cells. Journal of the American Society of Nephrology, 2014. 25(9): p. 2003-2015.

94. Rama, R., et al., Neuroprotective effect of Neuro-EPO in neurodegenerative diseases: "Alea jacta est". Neural regeneration research, 2019. 14(9): p. 1519.

95. Jang, W., et al., Safety and efficacy of recombinant human erythropoietin treatment of non-motor symptoms in Parkinson's disease. Journal of the neurological sciences, 2014. 337(1-2): p. 47-54.

96. Cevik, B., et al., Neuroprotective effects of erythropoietin on Alzheimer's dementia model in rats. Advances in Clinical and Experimental Medicine, 2017. 26(1): p. 23-29.

97. Miskowiak, K.W., et al., Recombinant human erythropoietin for treating treatment-resistant depression: a double-blind, randomized, placebo-controlled phase 2 trial. Neuropsychopharmacology, 2014. 39(6): p. 1399-1408.

98. Kim, H.Y., et al., Recombinant human erythropoietin in amyotrophic lateral sclerosis: a pilot study of safety and feasibility. Journal of clinical neurology, 2014. 10(4): p. 342-347.

99. Lauria, G., et al., Erythropoietin in amyotrophic lateral sclerosis: a multicentre, randomised, double blind, placebo controlled, phase III study. J Neurol Neurosurg Psychiatry, 2015. 86(8): p. 879-886.

100. Kim, S.H., Randomized, Double-blind, Safety and Efficacy of Recombinant Human Erythropoietin in Amyotrophic Lateral Sclerosis. 2019. 
101. Debeljak, N., P. Solár, and A.J. Sytkowski, Erythropoietin and cancer: the unintended consequences of anemia correction. Frontiers in immunology, 2014. 5: p. 563.

102. Nairz, M., et al., The pleiotropic effects of erythropoietin in infection and inflammation. Microbes and infection, 2012. 14(3): p. 238-246.

103. Chousterman, B.G. and M. Arnaud, Is there a role for hematopoietic growth factors during sepsis? Frontiers in Immunology, 2018. 9: p. 1015.

104. Kandasamy, K., et al., Erythropoietin reverses sepsis-induced vasoplegia to norepinephrine through preservation of $\alpha 1 D$-adrenoceptor mRNA expression and inhibition of GRK2-mediated desensitization in mouse aorta. Journal of cardiovascular pharmacology and therapeutics, 2016. 21(1): p. 100-113.

105. Nejat, R. and A.S. Sadr, Are losartan and Imatinib Effective Against SARS-CoV2 Pathogenesis? A Pathophysiologic-Based in Silico Study. chemRxiv preprint, 2020.

106. Kim, Y.-C., et al., Mechanism of erythropoietin regulation by angiotensin II. Molecular pharmacology, 2014. 85(6): p. 898-908.

107. Akimoto, T., et al., Erythropoietin modulates angiotensin II-or noradrenaline-induced Ca2+ mobilization in cultured rat vascular smooth-muscle cells. Nephrology Dialysis Transplantation, 2001. 16(3): p. 491-499.

108. Liu, Y., et al., Clinical and biochemical indexes from 2019-nCoV infected patients linked to viral loads and lung injury. Science China Life Sciences, 2020. 63(3): p. 364-374.

109. Saavedra, J.M., Brain angiotensin II: new developments, unanswered questions and therapeutic opportunities. Cellular and molecular neurobiology, 2005. 25(3-4): p. 485-512.

110. Kramár, E.A., J.W. Harding, and J.W. Wright, Angiotensin II-and IV-induced changes in cerebral blood flow: Roles of AT1 AT2, and AT4 receptor subtypes. Regulatory peptides, 1997. 68(2): p. 131138.

111. Elased, K.M., et al., Brain angiotensin-converting enzymes: role of angiotensin-converting enzyme 2 in processing angiotensin II in mice. Experimental physiology, 2008. 93(5): p. 665-675.

112. Alenina, N. and M. Bader, ACE2 in brain physiology and pathophysiology: Evidence from transgenic animal models. Neurochemical research, 2019. 44(6): p. 1323-1329.

113. Liu, Y., et al., Erythropoietin increases expression and function of transient receptor potential canonical 5 channels. Hypertension, 2011. 58(2): p. 317-324.

114. Harr, M.W. and C.W. Distelhorst, Apoptosis and autophagy: decoding calcium signals that mediate life or death. Cold Spring Harbor perspectives in biology, 2010. 2(10): p. a005579.

115. Tobu, M., et al., Erythropoietin-induced thrombosis as a result of increased inflammation and thrombin activatable fibrinolytic inhibitor. Clinical and applied thrombosis/hemostasis, 2004. 10(3): p. 225-232.

116. Demetz, G., et al., The influence of Erythropoietin on platelet activation, thrombin generation and FVII/active FVII in patients with AMI. Thrombosis journal, 2014. 12(1): p. 18.

117. Agarwal, R., Mechanisms and mediators of hypertension induced by erythropoietin and related molecules. Nephrology Dialysis Transplantation, 2018. 33(10): p. 1690-1698. 